\title{
Effects of Psychological Intervention on Pain and Mental State of Patients Underwent Total Thyroidectomy
}

\author{
Li Lijuan' ${ }^{1}$, Du Jing ${ }^{1}$, Li Chenguang ${ }^{1}$, Cui Zhao Xia ${ }^{2}$ and Ming Liu ${ }^{1 *}$ \\ ${ }^{1}$ Department of Breast Surgery, Affiliated hospital of Inner Mongolia Medical University, China \\ ${ }^{2}$ Shandong qilu pharmaceutical co. LTD, China
}

*Corresponding author: Ming Liu, Department of Breast Surgery, Affiliated hospital of Inner Mongolia Medical University, China

\begin{abstract}
ARTICLE INFO
Received: 慧 April 04, 2019

Published: 㓞 April 09, 2019

Citation: Li Lijuan, Du Jing, Li Chenguang, Cui Zhao Xia, Ming Liu. Effects of Psychological Intervention on Pain and Mental State of Patients Underwent Total Thyroidectomy. Biomed J Sci \& Tech Res 16(5)-2019. BJSTR. MS.ID.002923.
\end{abstract}

Keywords: Psychological Intervention; Total Thyroidectomy; Pain
ABSTRACT

Objective: To observe the effect of psychological intervention on pain and psychological state of patients underwent total thyroidectomy.

Methods: 104 patients who underwent total thyroidectomy in our hospital from March 2016 to March 2018 were selected and randomly divided into a study group and a control group, with 52 patients in each group. Psychological intervention was given to the patients in the study group and routine nursing was given to the control group. The changes of postoperative visual analogue scale (VAS), anxiety self-rating scale (SAS), depression self-rating scale (SDS), blood pressure and heart rate were observed.

Results: The VAS scores of the patients in the study group were $(2.31 \pm 0.12),(1.76 \pm 0.09)$, both lower than those in the control group [(3.95 \pm 0.20$),(3.42 \pm 0.24)] 24$ hours after surgery, with statistical significance $(\mathrm{P}<0.05)$. SAS [(38.38 2.25$)$ scores] and SDS [(39.34 \pm 2.04$)$ scores] in the study group were better than those in the control group [(48.71 \pm 2.32$),(49.44 \pm 3.2)$ scores], with statistically significant differences $(\mathrm{P}<0.05)$. Systolic blood pressure, diastolic blood pressure and heart rate $[(110.13 \pm 8.23) \mathrm{mmHg}(1 \mathrm{mmHg}=0.133 \mathrm{kpa}),(69.85 \pm 8.14) \mathrm{mmHg},(82.31 \pm 9.76)$ times / $\mathrm{min}]$ in the study group were better than those in the control group [(122.17 \pm 12.46$) \mathrm{mmHg},(90.08$ $\pm 7.23) \mathrm{mmHg}$, $(94.52 \pm 10.37)$ times $/ \mathrm{min}]$, and the differences were statistically significant $(\mathrm{P}<0.05)$.

Conclusion: psychological intervention for patients with total thyroidectomy can effectively reduce postoperative pain and improve psychological state of patients, which has clinical application value.

\section{Introduction}

In recent years, the incidence of thyroid cancer is on the rise globally year by year [1-2], among which the proportion of thyroid micro papillary cancer is gradual rise, while the treatment scheme is still not unified. Surgical resection is the most important means of treatment of thyroid papillary carcinoma [3]. The Futaba multifocal carcinoma in thyroid subtotal resection and one of the central lymph cleaning is the main operation. The prognosis of thyroid cancer post-surgery is usually good, but the total resection of thyroid will give patients both the psychological and physical pain. At the same time, due to the disease itself and the stimulation such as surgery, the patient is easy to have reactions of psychological stress after surgery such as anxiety. This may reduce the level of pain tolerance, increase patients' physical pain, affect patients with postoperative life quality and treatment effect [4-5]. In this study, the effect of psychological intervention on pain and psychological state of patients underwent total thyroidectomy was analyzed, and it is reported as follows.

\section{Materials and methods}

\section{General Information}

From March 2015 to March 2018, 1720 patients were operated on for thyroid cancer in our hospital, and 104 patients requiring total thyroidectomy for double-lobed multifocal carcinoma were randomly divided into a study group and a control group. 52 cases in the study group, male to female ratio: 26/26; age: 31 54 years old, mean (39.47 2.15) years old. 52 cases in the control group, male and female ratio of $26: 26$; average age (40.18 1.97) was between 32 and 55 years old. There was no significant difference in baseline data of age, gender and primary disease between the two groups $(\mathrm{P}$ $>0.05$ ), which was comparable. 


\section{Inclusion and Exclusion Criteria}

Inclusion criteria: those who were diagnosed with double lobe multifocal carcinoma of thyroid and met relevant clinical diagnostic criteria; All patients have signed the consent form for surgical treatment and nursing; no relevant contraindications [3-4]. Exclusion criteria: diagnosis with mental disorders and unwillingness to cooperate with psychological intervention.

\section{Methods}

The control group was given routine nursing, and relevant knowledge and information were given to the patients after admission. Preoperative preparations were carried out, and postoperative signs of the patients were monitored [6-7]. Psychological intervention was given to the study group, the specific way was as follows:

Psychological Intervention: Nursing staff actively communicated with patients. Psychological evaluation was carried out; and patients who were psychological instable received timely counseling; For example, for patients who worry about surgical pain and prognosis effect, the nursing staff will answer their questions with professional knowledge, including the introduction of surgical position, anesthesia method, anatomical characteristics, pain principle, etc., to alleviate the anxiety and tension of patients.

Cognitive Psychological Intervention: During hospitalization, relevant introduction such as the purpose of surgery, prognosis, etc. was given to the patients, so that patients and their families had a basic understanding of total thyroidectomy.
Posture Psychological Intervention: Preoperative guidance for patients with surgical posture practice. Put back cushion behind the patient during operation, keep the stability and comfort of the patient's head, to reduce the incidence of postoperative pain.

Pain Psychological Intervention: According to the postoperative pain to provide the patients guidance in correct way to get up, and reduction the head and neck activities, to reduce the postoperative pain.

\section{Curative Effect Observation Indexes}

Postoperative visual analogue scale (VAS) was used to evaluate postoperative pain in the two groups. $>3 \sim 6$ is moderate pain and tolerable. BBB 0 6 10 is classified as intense pain and unbearable [8]. After observing the situation before and after psychological intervention, the self-rating anxiety scale (SAS) and self-rating depression scale (SDS) were used to score: 50 was the barrier for anxiety or depression. 50 59 was for mild anxiety and depression. $>59 \sim 69$ were classified as moderate anxiety and depression.> 69 is classified as severe anxiety and depression [9]. The changes of SBP, DBP and HR in the two groups were observed, and the results were statistically analyzed [10].

\section{Statistical Methods}

SPSS 18.0 statistical software was used for data analysis. The measurement of data was shown as mean \pm standard deviation $(\mathrm{x} \pm \mathrm{s})$. And t test was used for comparison between the two groups. The rate of counting data indicated that the difference between the two groups was statistically significant by 2 test, $\mathrm{P}<0.05$ (Tables $1-3)$.

Table 1: Comparison of postoperative VAS pain scores between the two groups (see table 1, $\mathrm{x} \pm \mathrm{s}$ ).

\begin{tabular}{|c|c|c|c|c|}
\hline Group & Number & 1h after surgery & 24h after surgery & 48h after surgery \\
\hline Research Group & 52 & $4.15 \pm 0.37$ & $2.31 \pm 0.12$ & $1.76 \pm 0.09$ \\
\hline Control Group & 52 & $4.21 \pm 0.28$ & $3.95 \pm 0.20$ & $3.42 \pm 0.24$ \\
\hline
\end{tabular}

Note: $1 \mathrm{~h}(\mathrm{P}>0.05), 2448 \mathrm{~h}(\mathrm{P}<0.05)$.

Table 2: Comparison of SAS and SDS scores between the two groups after psychological intervention (see Table 2, $x \pm$ s).

\begin{tabular}{|c|c|c|c|}
\hline Group & Number & SAS & SDS \\
\hline Research Group & 52 & $38.38 \pm 2.25$ & $39.34 \pm 2.04$ \\
\hline Control Group & 52 & $48.71 \pm 2.32$ & $49.44 \pm 3.28$ \\
\hline
\end{tabular}

Note: Compared with the control group after psychological intervention, ${ }^{*} \mathrm{P}<0.05$; SAS: anxiety self-rating scale; SDS: depression self-rating scale.

Table 3: Comparison of postoperative blood pressure and heart rate between the two groups after psychological intervention (see Table 3,x s).

\begin{tabular}{|c|c|c|c|c|}
\hline Group & Number & SBP(mmHg) & DBP(mmHg) & HR(次 $/ \mathbf{m i n})$ \\
\hline Research Group & 52 & $110.13 \pm 8.23$ & $69.85 \pm 8.14$ & $82.31 \pm 9.76$ \\
\hline Control Group & 52 & $12.17 \pm 12.46$ & $90.08 \pm 7.23$ & $94.52 \pm 10.37$ \\
\hline
\end{tabular}

Note: $1 \mathrm{~mm} \mathrm{Hg}=0.133 \mathrm{kPa}$.

\section{The Results}

After psychological intervention, SBP, DBP and HR levels of patients in the research group were significantly better than those in the control group, with statistically significant differences $(\mathrm{P}<$ 0.05).

\section{Discussion}

Total resection of thyroid is the main surgical method of multifocal double leaf thyroid cancer. Because the whole thyroid resection patients could go through endocrine changes and stress reaction, this can lead to anxiety before and after surgery, 
postoperative secretion of adrenal cause heart rate, blood pressure, mental and physical discomfort, etc. All those can increase postoperative pain, influence the patient's quality of life and the later treatment [11-12]. In this study, a comprehensive and multimode psychological intervention was carried out on patients to reduce the factors that may aggravate the pain awareness of patients, reduce the pain perception of patients, and achieve a certain effect. Observe VAS pain score of the two groups of patients after surgery as a result, both groups of patients showed no significant differences in postoperative $1 \mathrm{~h}$ pain score. But the VAS score of $24 \mathrm{~h}$ and $48 \mathrm{~h}$ after surgery were (2.31-0.12) and (1.760.09 ) respectively, namely mild pain, and were significantly lower than the control group, suggests that psychological intervention can effectively reduce the thyroid surgery patients with pain. This result is related to psychological intervention before and after surgery. First, during hospitalization, the patient is informed about matters needing attention and knowledge of pain by medical staff. Thus, the patient can correctly avoid the internal and external factors that may cause postoperative pain under the guidance of relevant knowledge.

Secondly, during surgery nursing staff put cushions behind the patient's back to maintain the stability and comfort of the head, to reduce postoperative pain. Finally, after the operation, the medical staff guided the patient to get up, which effectively avoided the pain caused by head movements. The changes of patients' psychological states before and after the intervention were observed. There was no significant difference between the two groups before the intervention, and the SAS and SDS scores in the post-intervention study group were significantly better than those in the control group. When patients have anxiety or depression, the cholecystokinin will be activated, which can promote pain conduction and aggravate pain sensation. In addition, patients with physical pain usually show severe depression or generalized anxiety, that is, depression and anxiety are positively correlated with postoperative pain $[13,14]$. Therefore, psychological intervention can alleviate the patients' tension and anxiety with preoperative cognitive psychological intervention on the patients' diseases and surgeries, and improve the patients' mental state through psychological counseling, so that the patients can maintain a positive and optimistic mental state, thus reducing postoperative pain.

The blood pressure and heart rate of the two groups of patients after psychological intervention were observed. The blood pressure and heart rate of the study group were significantly lower than those of the control group, indicating that the adrenal secretion of the patients after psychological intervention gradually returned to normal. Blood pressure and heart rate began to decline, and the influence of surgical stress stimulation decreased. The above results proved that psychological intervention can improve the psychological state of patients in many ways, reduce postoperative pain, effectively reduce the stress stimulation of patients after total thyroidectomy, and improve the postoperative quality of life and effects of later treatments. In addition, medical staff can also use drugs to assist pain relief according to the specific situation, to further reduce the pain of patients. However, considering the possible adverse reactions caused by drugs, the principle of moderation should be followed $[15,16]$. In conclusion, psychological intervention for patients with total thyroidectomy can effectively reduce postoperative pain and improve psychological state.

\section{References}

1. Maniakas A, Davies L, Zafereo ME (2018) Thyroid Disease Around the world. Otolaryngol Clin North Am 51(3): 631-642.

2. Iniguez Ariza NM, Brito JP (2018) Management of Low-Risk Papillary Thyroid Cancer. Endocrinol Metab(seoul) 33(2): 185-194.

3. Miccoli P, Materazzi G, Macerola E, Bakkar S (2018) Tailrored surgery according to molecular analysis in differentiated thyorid carinomas 7(Suppl 1): S30-S33.

4. Song chuanyun (2012) Experience in postoperative pain care technology and psychological counseling [J]. China health nutrition 38(7): 20712072.

5. Chen yan (2012) Effect of nursing intervention on pain and negative emotions in patients undergoing thyroid surgery. Journal of modern Chinese medicine 21(32): 3637-3639.

6. Tang wen, Wang hongxiu, Tao jin (2014) Effect of nursing intervention on pain and stress response of patients undergoing thyroid surgery. Journal of community medicine 12(9): 75-76.

7. Song xiaomei, Tan lingfang, Deng aijuan (2012) Effect analysis of nursing intervention on postoperative pain in general surgery. Chinese contemporary medicine 19(12): 103-104.

8. Wang xuemei (2011) Study on the correlation between nursing intervention and postoperative pain after surgery. Chinese medical innovation 8(14): 14.

9. Peng lingjing (2014) Effect evaluation of nursing intervention in improving postoperative pain and negative emotions of patients undergoing thyroid surgery. Chinese journal of coal industry medicine, p. 17.

10.Xue chenglin, Yuan shuqing, Jiang hua (2012) Effect of nursing intervention on stress response of patients undergoing thyroid tumor surgery. Practical clinical medicine 13(5): 80-82.

11. Pan xueying (2013) Effect of individualized nursing intervention on anxiety of patients undergoing thyroid surgery. Liuzhou medical science 26(3): 2177-2179.

12. Maris, Liu Yang, Yu Wanyan (2014) Effect of nursing intervention on anxiety and stress in patients undergoing thyroid surgery. Chinese and foreign medical therapy 36(11): 150-152.

13. Chen Haiyan (2011) Effect of music intervention on patients with subtotal thyroidectomy. Contemporary nurse: academic edition 18(6): 142-143.

14. Liu Haiyan, Rao Aiqiong (2012) Effect of nursing intervention on pain and stress response of patients undergoing thyroid surgery. Clinical medical engineering 19(12): 2228-2229.

15. Tan Juju (2012) Application of whole-course nursing intervention in thyroidectomy. Contemporary nurses 19(3): 86-88.

16. Zhong Jing (2013) Effect of preoperative systematic nursing intervention on patients undergoing thyroid surgery. China health nutrition 26(4): 1848-1849. 


\section{ISSN: 2574-1241}

DOI: 10.26717/BJSTR.2019.16.002923

Ming Liu. Biomed J Sci \& Tech Res

(c) (P) This work is licensed under Creative

Submission Link: https://biomedres.us/submit-manuscript.php

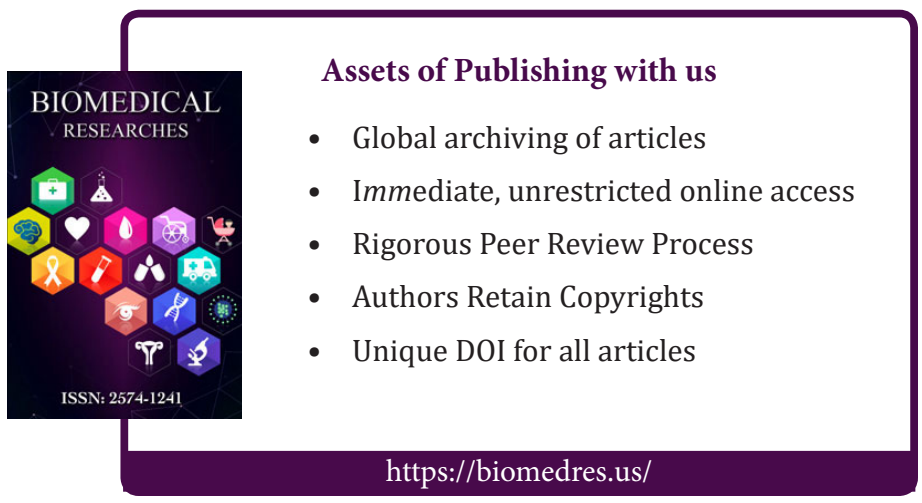

